\title{
Source imaging of deep-brain activity using the regional spatiotemporal Kalman filter
}

\author{
Laith Hamid ${ }^{\mathrm{a}, 1, *}$, Nawar Habboush ${ }^{\mathrm{a}, 1}$, Philipp Stern ${ }^{\mathrm{b}}$, Natia Japaridze $^{\mathrm{c}}$, Ümit $_{\text {Aydinn }}^{\mathrm{d}}$, \\ Carsten Wolters $^{\mathrm{d}}$, Jens Christian Claussen ${ }^{\mathrm{b}}$, Ulrich Heute ${ }^{\mathrm{e}}$, Ulrich Stephani ${ }^{\mathrm{c}}$, Andreas \\ Galka $^{\mathrm{a}}$, Michael Siniatchkin ${ }^{\mathrm{a}, 1}$ \\ ${ }^{a}$ Department of Medical Psychology and Medical Sociology, University of Kiel, D-24113 Kiel, Germany. \\ ${ }^{b}$ Institute of Experimental and Applied Physics, University of Kiel, 24098 Kiel, Germany. \\ ${ }^{c}$ Department of Neuropediatrics, University of Kiel, D-24098 Kiel, Germany. \\ ${ }^{d}$ Institute for Biomagnetism and Biosignalanalysis, University of Münster, D-48149 Münster, Germany. \\ ${ }^{e}$ Digital Signal Processing and System Theory Group, Faculty of Engineering, University of Kiel, D-24143 \\ Kiel, Germany. \\ ${ }^{f}$ Department of Child and Adolescent Psychiatry and Psychotherapy, Evangelisches Klinikum Bethel \\ gGmbH, 33617 Bielefeld, Germany.
}

\begin{abstract}
Background and objective: The human brain displays rich and complex patterns of interaction within and among brain networks that involve both cortical and subcortical brain regions. Due to the limited spatial resolution of surface electroencephalography (EEG), EEG source imaging is used to reconstruct brain sources and investigate their spatial and temporal dynamics. The majority of EEG source imaging methods fail to detect activity from subcortical brain structures. The reconstruction of subcortical sources is a challenging task because the signal from these sources is weakened and mixed with artifacts and other signals from cortical sources. In this proof-of-principle study we present a novel EEG source imaging method, the regional spatiotemporal Kalman filter (RSTKF), that can detect deep brain activity.

Methods: The regional spatiotemporal Kalman filter (RSTKF) is a generalization of the spatiotemporal Kalman filter (STKF), which allows for the characterization of different regional dynamics in the brain. It is based on state-space modeling with spatially heterogeneous dynamical noise variances, since models with spatial and temporal ho-
\end{abstract}

\footnotetext{
* Corresponding author

Email address: laithala jeel@gmail . com (Laith Hamid)

${ }^{1}$ Both authors contributed equally to this work
} 
mogeneity fail to describe the dynamical complexity of brain activity. First, RSTKF is tested using simulated EEG data from sources in the frontal lobe, putamen, and thalamus. After that, it is applied to non-averaged interictal epileptic spikes from a presurgical epilepsy patient with focal epileptic activity in the amygdalo-hippocampal complex. The results of RSTKF are compared to those of low-resolution brain electromagnetic tomography (LORETA) and of standard STKF.

Results: Only RSTKF is successful in consistently and accurately localizing the sources in deep brain regions. Additionally, RSTKF shows improved spatial resolution compared to LORETA and STKF.

Conclusions: RSTKF is a generalization of STKF that allows for accurate, focal, and consistent localization of sources, especially in the deeper brain areas. In contrast to standard source imaging methods, RSTKF may find application in the localization of the epileptogenic zone in deeper brain structures, such as mesial frontal and temporal lobe epilepsies, especially in EEG recordings for which no reliable averaged spike shape can be obtained due to lack of the necessary number of spikes required to reach a certain signal-to-noise ratio level after averaging.

Keywords: deep sources, dynamical inverse solution, EEG, EEG inverse problem, EEG source imaging, Electroencephalography, epilepsy, epileptiform activity, Kalman filter, LORETA, RSTKF, source reconstruction, spatiotemporal Kalman filter, state space, STKF, subcortical sources.

\section{Introduction}

Eelectroencephalography (EEG) is a non-invasive electrophysiological method which measures the time series of electric potential differences on the surface of the head that are caused by the summation of neuronal depolarization shifts on postsynaptic membranes of a large number of neurons. Due to its high temporal resolution (The maximum sampling rate of modern EEG systems is in the $\mathrm{KHz}$ range), EEG can be used to study brain function and track temporal brain activity. In order to increase the spatial resolution of EEG, source imaging uses these surface measurements and estimates the generators of electrical activity in the brain. EEG source imaging has found numer- 
ous applications in cognitive brain research, neuropsychiatry, clinical neurology, and neuropediatrics [1-4].

The problem of source reconstruction from surface EEG becomes particularly challenging when changes of electrical activity on the scalp result from neuronal depolarization in deep brain structures, since deep sources generate only weak surface signals and are often masked by the activity of strong cortical sources. The localization of deep sources can have many applications in clinical and cognitive research, e.g., in understanding normal and pathological brain function as well as studying brain networks [5-7]. An example of a deep source that is important for clinical application is epileptic activity in the hippocampus, since hippocampal pathology is the most frequent cause of the mesial temporal lobe epilepsy which may be successfully treated by surgery [8].

Regarding the solution of the EEG inverse problem, we will limit our discussion here to distributed-source methods (for an overview please refer to [9-12]). In these methods the activity of the whole brain is estimated for each time point using the whole scalp EEG. In order to obtain a unique solution to the EEG inverse problem, standard EEG source imaging methods impose constraints on the solution space using, e.g., a penalty function or a Bayesian framework [13]. Among the standard source reconstruction methods, low-resolution brain electromagnetic tomography (LORETA) is one of the most frequently used approaches [14]. This method applies spatial smoothness as a constraint to obtain a unique solution to the EEG inverse problem and penalizes non-smooth solutions. Standard methods, such as minimum norm and standardized LORETA (sLORETA) [15], were shown to be problematic for the localization of deep sources especially when cortical sources masked the deeper activity or in the presence of multiple sources [12]. Additionally, the majority of the standard source reconstruction methods localize the sources at a specific time point without making use of the source reconstruction results of the previous time points, i.e., they are static or instantaneous methods. The temporal information of the EEG recordings includes useful information about the locations of the sources and the interactions between them.

A dynamical inverse solution avoids the shortcomings of static methods by taking the information from the source reconstructions at the previous time points into account when reconstructing the sources at a certain time point [16-18]. State-space 
modeling is a flexible modeling approach to implement a dynamical inverse solution from EEG time series [19]. A state-space model consists of two equations that describe the spatiotemporal dynamics in the brain and the measurement process respectively. The reconstruction of the brain's primary current density, which is defined as the system's state, is transformed into a problem of state estimation. The Kalman filter [20] provides the optimal linear solution for the problem of state estimation. If Gaussian noise is assumed, the Kalman filter is optimal in the sense that it provides the minimum mean square error estimates of the state variables. If the Gaussianity condition is not fulfilled, the Kalman filter is still the best linear minimum mean square error estimator [21]. Modified versions of the Kalman filter have found many applications in inverse modeling, especially in high-dimensional problems of climate and ocean modeling [22,-24]. However, the estimation of parameters and the propagation of huge covariance matrices become infeasible in case of high dimensionality. Instead of the full Kalman filter, filters that are based on strong simplifications of the deterministic model and low-rank approximations of the covariance matrices are usually employed to solve the problem of high dimensionality [24-26].

The spatiotemporal Kalman filter (STKF) [18] is a dynamical solution of the highdimensional EEG inverse problem that is based on a modification of the traditional Kalman filter. This method and its adaptive forms belong to the family of distributedsource inverse methods. Within the framework of STKF, the state-space model parameters are estimated via maximum likelihood (ML). In addition to spatial smoothness, STKF uses temporal smoothness to obtain a unique solution to the EEG inverse problem. The spatiotemporal smoothness constraint can be implemented by appropriate design of the parameter matrices within the state-space model. Since both LORETA and STKF use spatial smoothness constraints, we consider it useful to compare their performance, in order to ascertain the additional advantages of temporal smoothness in the STKF model. In previous work, the original STKF model was found to be superior to LORETA with respect to the localization of sources of alpha rhythms, epileptiform discharges, and focal seizures from EEG recordings [27,-32]. When applied to localize sources of a focal seizure from an EEG recording, STKF showed more accurate and consistent localizations of the seizure onset, compared to LORETA [27]. Addi- 
tionally, STKF produced more accurate source reconstructions from small numbers of electrodes (9 and 19 electrodes), compared to LORETA [30].

The STKF method is based on a linear state-space model with spatially and temporally constant parameters. The performance of the method depends on the dynamical model, and the accuracy of the results could be improved with more accurate and more physiological dynamical models. An important limitation of this model is that it assumes spatial homogeneity of the brain, in contradiction to what is known about functional specialization and modularity of the brain. In order to mitigate the abovementioned limitation of the original STKF model, namely the spatial homogeneity of the model parameters, and to permit multiple areas in the brain to have their own dynamics, the state-space model was modified in this work to describe different brain regions using different dynamical noise variance parameters. Based on the previous experience of the authors, the dynamical noise variance parameter was judged to be the most influential one among the parameters of state-space models, and the stochastic part of the dynamical model was extended accordingly. This new approach is named the regional spatiotemporal Kalman filter (RSTKF).

The aim of this work is the extension of the dynamical approach of spatiotemporal Kalman filtering (STKF) by allowing for region-specific dynamics in the state-space model of the brain. The hypothesis here is that this extension permits the correct localization of active subcortical areas in the brain from surface EEG, since each one of these areas will be allowed to have its own dynamics. Additionally, we expect this extension to improve the accuracy and spatial resolution of EEG source reconstruction results, due to the regional specification of model parameters. In this paper, we will test the RSTKF using simulated as well as clinical EEG data. We will concentrate on single-source scenarios and the clinical application of the localization of the epileptogenic zone from EEG in presurgical evaluation. The results of RSTKF will be compared to those of LORETA and STKF. We expect RSTKF to outperform LORETA and STKF with respect to accuracy and spatial resolution of the source reconstruction results. 


\section{Materials and Methods}

In the first section, the regional spatiotemporal Kalman filter algorithm is first described. After that, the setup for source imaging of simulated EEG data is described. In the last section, the setup for source imaging of clinical EEG data of epileptic spikes is described. For these analyses, the EEG source imaging results from LORETA, STKF, and RSTKF are compared. In order to avoid the inversion of high-dimensional matrices, the alternative implementation of LORETA from [18, 33] was used in this paper. Additionally, the Akaike Bayesian Information Criterion (ABIC) was used to estimate the regularization parameter, as in [18, 33]. The preprocessing and source imaging was performed in MATLAB (Mathworks, MATLAB 7.12, R2011a). The visualization of EEG source imaging results was done using the open-source Fieldtrip toolbox [34]. The whole procedure of preprocessing, head modeling, source imaging, and validation is summed up in Fig. 1 .

[Figure 1 about here.]

\subsection{The regional spatiotemporal Kalman filter (RSTKF)}

Until now, the model parameters of the STKF were non-adaptive with respect to both time and space. Due to the brain's modularity and functional specialization, we believe that introducing non-homogeneity in space, e.g. in lobar or sub-lobar levels, is essential to describe the different processes that occur in different parts of the human brain. The distinction of different regions in the brain was introduced into the LORETA constraint in [35]. Assuming spatial homogeneity of the parameters was only a strong assumption with the purpose of making parameter estimation feasible. The RSTKF uses a 3D voxel grid whose grid points are labeled according to the brain region. This classification or labeling process is non-trivial, especially for individual anatomies. According to Wold's decomposition theorem [36], a very sophisticated deterministic model which is accompanied by a simple stochastic model, can be replaced by a fairly simple deterministic model in addition to a sophisticated stochastic model in the description of the system's dynamics by a state-space model [19, 36]. 
In this paper, a simple deterministic model of the dynamics was used in addition to a more detailed model of the stochastic term. For each brain region, a separate dynamical noise variance is assigned and the values of these dynamical noise variances are learned from the data using ML parameter estimation. Mathematically, the RSTKF uses the same equations and model parameters that are used by the STKF, except for the modified description of the dynamical noise term $\boldsymbol{\eta}_{t}$ and its covariance matrix $\boldsymbol{\Sigma}_{\eta_{L}}$.

The measurement of EEG time series is modeled as follows:

$$
\mathbf{y}_{t}=\mathbf{K} \mathbf{j}_{t}+\boldsymbol{\epsilon}_{t}
$$

Here, $\mathbf{y}_{t}$ is the $N_{y} \times 1$ vector of measurements at time $t$, where $N_{y}$ represents the number of EEG electrodes. The $N_{y} \times N_{j}$ matrix $\mathbf{K}$ is the lead field matrix, or gain matrix. This matrix includes the contribution of each voxel to each EEG electrode. $\mathbf{J}_{t}$ is the $N_{j} \times 1$ vector of primary current density values in the brain. The brain is represented as a volumetric 3D voxel grid, and each grid point is the location of three current dipoles. Each one of these three current dipoles points to one of the three Cartesian coordinates, in the $x, y$, or $z$ directions. With $N_{v}$ denoting the number of grid points, the number of current dipoles $N_{j}$ equals $3 \times N_{v}$. This grid is then further divided into $N_{r}$ regions, where $N_{r}$ is the number of modeled regions in the brain.

One solution to the high dimensionality of the EEG inverse problem is to use a modification of the standard Kalman filter as implemented in the STKF approach [18]. In order to tackle the high-dimensional EEG inverse problem, the Kalman filter is transformed from a fully-coupled state-space into a weakly-coupled system, which only retains nearest-neighbor coupling. This is done by using the second-order discrete Laplacian operator to decouple the state-space model, preserving only nearest-neighbor interactions. This transformation is called spatial whitening [18, 37]:

$$
\tilde{\mathbf{j}}_{t}=\mathbf{L} \mathbf{j}_{t}
$$

The $N_{j} \times N_{j}$ Laplacian matrix $\mathbf{L}$ is the discrete second-order spatial Laplacian operator and approximates the second spatial derivative. In order to calculate the Laplacian matrix, the neighboring grid points for each grid point $\mathrm{v}$ are found and the matrix of 
neighbors $\Omega$ is constructed according to:

$$
\boldsymbol{\Omega}_{\hat{v} v}= \begin{cases}1 & \text { if } v \text { is a neighbor of } v \\ 0 & \text { otherwise }\end{cases}
$$

Only 6 neighbors in the positive and negative $x, y$, and $z$ directions are considered in the computation of the Laplacian matrix. Then the following equation is used to compute the Laplacian matrix:

$$
\mathbf{L}=\left(\mathbf{I}_{N_{v}}-\frac{1}{6} \boldsymbol{\Omega}\right) \otimes \mathbf{I}_{3} .
$$

After that, a low-dimensional Kalman filter is calculated at each grid point, the state of which is influenced by the average value of the current densities of the neighboring grid points. For the autoregressive (AR) model of order 1, the state dimension $N_{s t}$ is 3 and the dynamical model can be written as follows

$$
\tilde{\mathbf{j}}_{v, t}=\mathbf{A}_{L} \tilde{\mathbf{j}}_{v, t-1}+\mathbf{B}_{L} \tilde{\mathbf{u}}_{v, t}+\tilde{\eta}_{t}
$$

The $3 \times 3$-dimensional local state transition matrix $\mathbf{A}_{L}$ characterizes the voxel's selfdynamics, whereas the contribution of the neighboring voxels to the voxel's current density is described by the $3 \times 3$-dimensional local input matrix $\mathbf{B}_{L}$. The local extrinsic control input vector $\tilde{\mathbf{u}}_{(v, t)}$ consists of the average value of the previously computed current densities of each voxel's immediate neighbors.

$$
\tilde{\mathbf{u}}_{v, t}=\frac{1}{6} \sum_{v \in \mathscr{N}(v)} \tilde{\mathbf{j}}_{v, t-1}
$$

Finally, the $3 \times 3$-dimensional local dynamical noise covariance matrix $\boldsymbol{\Sigma}_{\tilde{\eta} L}$ and the $\mathbf{A}_{L}$ and $\mathbf{B}_{L}$ matrices [38] are defined by

$$
\mathbf{A}_{L}=a_{1} \mathbf{I}_{3}, \quad \mathbf{B}_{L}=b \mathbf{I}_{3}, \quad \Sigma_{\tilde{\eta} L}=\sigma_{\tilde{\eta}}^{2}(v) \mathbf{I}_{3}
$$

144 Here, $\sigma_{\tilde{\eta}}^{2}(v) \in \sigma_{\eta 1}{ }^{2}, \ldots, \sigma_{\eta N r^{2}}{ }^{2}$, with $N_{r}$ being the number of modeled regions in the 145 brain.

In [32] the reformulation of the standard autoregressive model of order 2 into an augmented state-space model, in which the standard second-order AR component is reformulated as a first-order one, is explained. In the new model, $N_{s t}$ equals 6 and the 
equation of the dynamical model becomes [32]:

$$
\left[\begin{array}{l}
\tilde{\mathbf{j}}_{v, t} \\
\tilde{\tilde{\mathbf{j}}}_{v, t}
\end{array}\right]=\mathbf{A}_{L}\left[\begin{array}{l}
\tilde{\mathbf{j}}_{v, t-1} \\
\tilde{\tilde{\mathbf{j}}}_{v, t-1}
\end{array}\right]+\mathbf{B}_{L}\left[\begin{array}{c}
\tilde{\mathbf{u}}_{v, t} \\
\mathbf{0}
\end{array}\right]+\left[\begin{array}{l}
\tilde{\eta}_{t} \\
\mathbf{0}
\end{array}\right] .
$$

The $3 \times 1$-dimensional vector $\overline{\tilde{\mathbf{j}}}_{v, t}$ is the predicted value of $\overline{\tilde{\mathbf{j}}}_{v, t+1}$. As for the model parameter matrices, the $6 \times 6$-dimensional local state transition matrix $\mathbf{A}_{L}$ is constructed as follows:

$$
\mathbf{A}_{L}=\left[\begin{array}{cc}
a_{1} \mathbf{I}_{3} & a_{2} \mathbf{I}_{3} \\
\mathbf{I}_{3} & \mathbf{0}
\end{array}\right] .
$$

Likewise, the $6 \times 6$-dimensional local input matrix $\mathbf{B}_{L}$ is constructed as follows:

$$
\mathbf{B}_{L}=\left[\begin{array}{cc}
b \mathbf{I}_{3} & \mathbf{0} \\
\mathbf{0} & \mathbf{0}
\end{array}\right] \text {. }
$$

Finally, the local dynamical noise covariance matrix $\Sigma_{\tilde{\eta}_{L}}$ can be written as [38]:

$$
\Sigma_{\tilde{\eta}_{L}}=\left[\begin{array}{cc}
\sigma_{\tilde{\eta}}^{2} \mathbf{I}_{3} & \mathbf{0} \\
0 & 0
\end{array}\right]
$$

In the above-mentioned dynamical model, autoregressive moving average (ARMA) type modeling consists of autoregressive (AR) and moving average (MA) parts. In principle, each of these parts could do the modeling alone, so there is a kind of redundancy between them. Just for the sake of flexible and parsimonious modeling, both parts are used. Variance parameters correspond to the MA part. Due to this redundancy, it seems risky to allow regional dependence both for AR and MA parts; this may lead to overfitting. A similar situation, namely time-dependent variance, suffers from the same problem.

The lead field matrix $\mathbf{K}$ is now replaced by the Laplacianized lead field matrix $\tilde{\mathbf{K}}$ which is calculated as follows:

$$
\tilde{\mathbf{K}}=\mathbf{K L}^{-1}
$$

We should also mention that the measurement noise covariance matrix $\Sigma_{\varepsilon}$ is assumed to have the following structure:

$$
\Sigma_{\varepsilon}=\sigma_{\varepsilon}^{2} \mathbf{I}_{3}
$$


In the following, $N_{s t}$ represents the dimension of the state for each grid point. In the prediction phase, the STKF uses the dynamical model to make predictions about the current density values at the next time point:

$$
\tilde{\mathbf{j}}_{v, t \mid t-1}=\mathbf{A}_{L} \tilde{\mathbf{j}}_{v, t-1 \mid t-1}+\frac{1}{6} \mathbf{B}_{L} \sum_{v \in \mathscr{N}(v)} \tilde{\mathbf{j}}_{\hat{v}, t-1 \mid t-1}
$$

Now, the $N_{s t} \times N_{s t}$-dimensional local state prediction error covariance matrix is calculated from:

$$
\boldsymbol{\Sigma}_{\tilde{j}_{v, t \mid t-1}}=\mathbf{A}_{L} \boldsymbol{\Sigma}_{\tilde{j}_{v, t-1 \mid t-1}} \mathbf{A}_{L}^{\top}+\boldsymbol{\Sigma}_{\tilde{\eta}_{L}}
$$

After that, the state predictions for all grid points are used to compute a measurement prediction

$$
\mathbf{y}_{t \mid t-1}=\tilde{\mathbf{K}} \tilde{\mathbf{j}}_{t \mid t-1} .
$$

The difference between the actual and predicted measurement values constitutes the $N_{j} \times 1$-dimensional measurement prediction error, residual, or innovation

$$
\mathbf{r}_{y, t}=\mathbf{y}_{t}-\mathbf{y}_{t \mid t-1}
$$

${ }_{154}$ Let $\tilde{\mathbf{K}}_{v}$ denote the $N_{y} \times 3$-dimensional sub-matrix within the lead field matrix $\tilde{\mathbf{K}}$ that corresponds to grid point $v$. Since $\tilde{\mathbf{K}}$ is a global matrix linking all positions in the brain with all EEG electrodes, therefore it depends very much on the spatial position and, thereby, on the region.

Then, the innovation covariance matrix is calculated as follows:

$$
\boldsymbol{\Sigma}_{\mathbf{r}_{y, t}}=\sum_{v} \tilde{\mathbf{K}}_{v} \boldsymbol{\Sigma}_{\tilde{j}_{v, t \mid t-1}} \tilde{K}_{v}^{\top}+\Sigma_{\mathcal{\varepsilon}} .
$$

After calculating the innovation and its covariance matrix, the $N_{s t} \times N_{y}$-dimensional local Kalman gain matrix is calculated using:

$$
\mathbf{G}_{v, t}=\boldsymbol{\Sigma}_{\tilde{j}_{v, t \mid t-1}} \tilde{\mathbf{K}}_{v}^{\top} \boldsymbol{\Sigma}_{\mathbf{r}_{y, t}}^{-1} .
$$

After that, the $N_{s t} \times 1$-dimensional local filtered state estimate is computed from:

$$
\tilde{\mathbf{j}}_{v, t \mid t}=\tilde{\mathbf{j}}_{v, t \mid t-1}+\mathbf{G}_{v, t} \mathbf{r}_{y, t} .
$$


The local $N_{s t} \times N_{s t}$ state estimation error covariance matrix is obtained from:

$$
\boldsymbol{\Sigma}_{\tilde{j}_{v, t} \mid t}=\left(\mathbf{I}_{N_{s t}}-\mathbf{G}_{v, t} \tilde{\mathbf{K}}_{v}\right) \boldsymbol{\Sigma}_{\tilde{j}_{v, t} \mid t-1} .
$$

The final vector of state estimates and its error covariance matrix are transformed back from the weakly-coupled state-space into the fully-coupled state-space using the following equations:

$$
\begin{gathered}
\mathbf{j}_{t}=\mathbf{L}^{-1} \tilde{\mathbf{j}}_{t} . \\
\boldsymbol{\Sigma}_{j_{v, t \mid t}}=\mathbf{L}^{-1} \Sigma_{\tilde{j}_{v, t \mid t}} \mathbf{L}^{-1 \top} .
\end{gathered}
$$

The Kalman filter calculates the state estimate and its error covariance matrix. The parameter matrices of the state-space model, however, need to be estimated before calculating the output of the Kalman filter.

In this paper, the optimal parameters of the state-space model are obtained using minimization of the Akaike Information Criterion (AIC) [39], which corresponds to Maximum-Likelihood estimation with an additional constraint favoring parsimonious models. The AIC is defined by

$$
\operatorname{AIC}\left(\theta_{K F}\right)=-2 \mathscr{L}\left(\theta_{K F}\right)+2 N_{K F} .
$$

Here, $\boldsymbol{\theta}_{K F}$ denotes the vector of model parameters of the RSTKF. $\mathscr{L}\left(\theta_{K F}\right)$ denotes the log-likelihood, i.e., the probability that the data were generated by a model with parameter vector $\boldsymbol{\theta}_{K F} . N_{K F}$ denotes the dimension of the parameter vector $\boldsymbol{\theta}_{K F}$. Now for the RSTKF, the following parameter vector was used:

$$
\theta_{K F}=\left[a_{1}, a_{2}, b, \sigma_{\tilde{\eta}_{1}}^{2}, \ldots, \sigma_{\tilde{\eta}_{N r}}^{2}, \sigma_{\varepsilon}^{2}\right]^{\top}
$$

The parameter vector was divided into three groups, and at the beginning of the parameter estimation procedure these groups were individually optimized. The dynamical AR parameters $a_{1}, a_{2}, b$ constitute the first group. The second group includes the dynamical noise variance parameters $\sigma_{\tilde{\eta}_{1}}^{2}, \ldots, \sigma_{\tilde{\eta}_{N r}}^{2}$ and the third and final group contains solely the measurement noise variance parameter $\sigma_{\varepsilon}^{2}$. This approach alleviates the problems of slow convergence, parameter redundancy at the optimal point, and ill-conditioned 
parametrization of the state-space model. After the group-wise optimization, all parameters should be jointly optimized [19].

The RSTKF is used to evaluate the AIC according to equation 2.1 and the following definition of the log-likelihood:

$$
\mathscr{L}\left(\theta_{K F}\right)=-\frac{1}{2} \sum_{t=1}^{T}\left(\log \left|\Sigma_{\mathbf{r}_{y, t}}\right|+\mathbf{r}_{y, t}{ }^{\top} \Sigma_{\mathbf{r}_{y, t}}^{-1} \mathbf{r}_{y, t}\right)+N_{y} \log (2 \pi) .
$$

Here, $T$ is the number of time points in the data and $N_{y}$ is the number of electrodes. All EEG data and all source regions are employed by a single modeling approach which yields a single value for the AIC, for each given set of model parameters.

Our approach of parameter fitting is an application of classical maximum-likelihood based on the innovation likelihood [19, 40]. Two optimization algorithms were used to minimize the AIC. The first method is the Broyden-Fletcher-Goldfarb-Shanno (BFGS) method, a quasi-Newton approach, as implemented in MATLAB's Optimization Toolbox. The second method is the Nelder-Mead simplex algorithm, which was also used as it is implemented in MATLAB. During the analyses, either the BFGS method alone or an iteration of BFGS and simplex methods was used for the optimization [18, 32]. This iteration of the two methods helps when the BFGS faces numerical problems. Based on the authors' experience, numerical problems with BFGS happen only rarely for this application of the methods. For this application, the gradient and Hessian of the cost function for the BFGS method are estimated numerically by the MATLAB function. Cross validation would be used in order to avoid overfitting, but AIC has its own penalty term against overfitting. Besides, even with regional parameters our model is still very parsimonious (unless we would choose a high number of regions, which is infeasible).

\subsection{Source imaging of simulated data}

The first step in the development of the regional spatiotemporal Kalman filter (RSTKF) was the use of simulated EEG data from single sources with known locations, orientations and strengths. Then the depth of these sources was increased and the number of modeled brain regions was also increased to investigate the possible problems in the 
source localization and parameter estimation due to the increase in the source depth or the number of model parameters. The simulations in this section were performed using the standard realistically-shaped three-compartment head model from the Neuroscan Curry software (Compumedics Neuroscan, version 7.0) which is computed using the boundary element method (BEM). The source space was defined using a 3D volumetric grid with a spacing of $5 \mathrm{~mm}$. For the simulation, the grid had a different grid spacing compared to the one used for the inverse solution to avoid an inverse crime. The BEM head model consisted of three compartments: inner skull space, outer skull, and BEM skin. 3858 nodes described the inner skull space, which includes the brain and the cerebrospinal fluid (CSF), and its conductivity value was set to $0.33 \mathrm{~S} / \mathrm{m}$. The second compartment, the outer skull was built from 2681 nodes and skull compartment conductivity was set to $0.0042 \mathrm{~S} / \mathrm{m}$. Finally, the BEM skin surface had 1504 nodes and skin compartment conductivity was set to $0.33 \mathrm{~S} / \mathrm{m}$.

In the first simulation, a single dipole was simulated and the source signal was generated in the theta range with a frequency of $4.8 \mathrm{~Hz}$ and a sampling rate of 256 $\mathrm{Hz}$ using an autoregressive model of order two. The orientation of the dipole was randomly rotating in the yz-plane around the $\mathrm{x}$-axis, which is perpendicular to the ears. An example of the randomly rotating dipole and its source signal is shown in Fig. 2. This simulation scheme was chosen because we hypothesized that the choice of a rotating dipole forces the Kalman filter to track the source, since the EEG signal is highly sensitive to the orientation of the sources. Additionally, the dynamical nature of the Kalman filter makes it easier to assume a deep source that is changing its orientation than a group of active cortical sources that span the whole cortex. Three different simulated EEG datasets were generated using single dipoles with increasing depth; the first dipole was placed in the left frontal lobe, the second one was placed in the left putamen, and the third one was placed in the left thalamus. In each of these cases, the simulated source signal was multiplied with the lead field matrix and $-80 \mathrm{~dB}$ white Gaussian measurement noise was added to produce a 32-channel EEG dataset. The sources in the frontal lobe, putamen, and thalamus resulted in signal to noise ratio (SNR) values of 45.77, 44.05, and 42.62, respectively. The resulting EEG datasets are depicted in Fig. 3 
[Figure 2 about here.]

[Figure 3 about here.]

In order to test the RSTKF approach, EEG source imaging was performed on the simulated EEG data from the frontal lobe, putamen, and thalamus using LORETA, STKF, and RSTKF(7). Here, RSTKF(7) denotes a 7-region RSTKF. Based on [5, 6], we divided the source space into the thalamus, hippocampus, putamen, and caudate nucleus. Additionally, we added the cerebellum and what was left of the left and right hemispheres as the last three regions. The definition of the subcortical regions did not distinguish left and right structures. We decided to model the cerebellum because of its connection to the cerebellar cortex and its involvement in sensorimotor and cognitive functions such as language and attention [41]. The LORETA, STKF, and RSTKF(7) analyses were performed using the standard BEM head model from CURRY7 (Neuroscan, Compumedics) and the brain was discretized using a 3D volumetric grid with $7 \mathrm{~mm}$ grid spacing.

The Euclidean distances (in $\mathrm{mm}$ ) between the simulated and estimated current density maxima were calculated for all simulated sources and source imaging methods. No repetition, averaging or cross validation were used for the evaluation of the results via Euclidean distance.

Additionally for the case of the thalamic source, which was the deepest source in our simulations, the number of modeled regions was varied between two and seven regions to test the performance of $\operatorname{RSTKF}(7)$. In the first analysis, two regions were defined with one region in the thalamus and another one to include the rest of the brain, then the analysis was performed for the case of three regions consisting of the thalamus, left hemisphere, and right hemisphere. After that, the hippocampus was added as the fourth region. The cerebellum was added in the 5-region RSTKF, while the putamen and caudate nucleus were added for the 6- and 7-region RSTKF, respectively. The RSTKF(2) and RSTKF(3) were run using a 3D volumetric grid with $14 \mathrm{~mm}$ spacing. In all of the aforementioned cases, a $7 \mathrm{~mm}$ grid spacing was used for the $3 \mathrm{D}$ volumetric grid that describes the source space. In this work, a choice needed to be made between using $5 \mathrm{~mm}$ grid spacing to represent small or longitudinal subcortical areas 
with enough grid points or to dilate the segmented subcortical areas in order to make their representation with $7 \mathrm{~mm}$ grid spacing feasible. The latter approach was used in this paper. The classical optimization scheme was used for parameter estimation and the dynamical model was an autoregressive model of order 2 for all the analyses in this section.

\subsection{Source imaging of single-trial spikes from a presurgical patient of epilepsy}

The EEG data was recorded from a teenage female patient during presurgical evaluation. The patient was diagnosed with a drug-resistant, symptomatic mesial temporal lobe epilepsy (TLE) due to a hippocampal sclerosis in the left hemisphere. The diagnosis was performed according to the clinical guidelines described in [42] . The patient suffered from daily psychic auras and complex focal seizures. Prior to surgery, the EEG showed inter-ictal and ictal changes in the left temporal region. The patient's MRI showed a hippocampal sclerosis, a thickening of the left amygdala and caput hippocampi. Finally, the neuropsychological tests indicated a dysfunction of the left temporal lobe. Based on this diagnosis, the patient was operated with selective resection of amygdala and the hippocampus. After the surgery, the patient became seizure-free (outcome Engel $\mathrm{Ib}[43]$ ). The data was fully anonymized. According to paragraph 15 of the Rules of Professional Practice of the Medical Association of Schleswig-Holstein, we do not need an ethics vote for the analysis of fully-anonymized data. The study was conducted according to the Declaration of Helsinki (current version, 1996) on biomedical research involving human subjects (Tokyo amendment). Parents or legal guardians of participants were informed about the research purposes and gave verbal informed consent, which was not recorded, to keep the procedure anonymous.

The presurgical EEG was registered with a Nihon Kohden system (http://www.nihonkohden.com). 40 electrodes were placed according to the 10/10 system, including additional temporal electrodes. A sampling frequency of $500 \mathrm{~Hz}$ was used and the impedance was kept below $10 \mathrm{kOhm}$. First, five artifact-free 4-second EEG segments were marked and visually selected, each of which contained a single spike in the middle of the interval. In order to reduce the computational time expense, only 0.8 seconds of each segment were chosen such that the spike's peak always occurred at 0.5 seconds. The pre-spike 
interval was longer than the post-spike interval by 0.2 seconds in order to allow for a transient of the Kalman filter. The waveforms of the mean global field power (MGFP) for the five spikes are depicted in Fig. 4 with markers and voltage maps at the spike peaks. The spike onset was determined from the spike component extracted using principal component analysis (PCA). After that, a notch filter (at $50 \mathrm{~Hz}$, width of $2 \mathrm{~Hz}$ ) was applied to remove the power-supply artifact. Additionally, a high pass filter with a cut-off frequency of $0.53 \mathrm{~Hz}$ was used to remove any drifts. The filters were forward filters of Butterworth type. Additionally, the EEG data was re-referenced to the common average reference before applying LORETA, STKF or RSTKF. For every spike, the SNR was calculated as described in [28, 44]. The (negative) peak to (positive) peak amplitude of the spike, which is defined to fall within $175 \mathrm{~ms}$ around the spike peak, at the dominant electrode was calculated and divided by the root mean square (rms) value of the background activity of the same electrode, which occurs in the $300 \mathrm{~ms}$ preceding the spike duration (175 $\mathrm{ms}$ around the spike peak).

For head modeling, again the standard 3-compartment BEM head model from CURRY7 was used. The source space was defined using a 3D volumetric grid with $7 \mathrm{~mm}$ resolution. CURRY7 was also used to partition the source space into 7 regions by generating volumetric grids of every region and using them for labeling the points in the source space. The 7 regions included the thalamus, amygdalo-hippocampal region, putamen, caudate nucleus, cerebellum, the remainder of the left hemisphere, and the corresponding volume of the right hemisphere. The subcortical regions here were not divided into left and right structures. The dilation of subcortical structures was also used here in order to represent each of these structures with enough points in the $7 \mathrm{~mm}$ grid.

[Figure 4 about here.]

The current density estimates were computed using LORETA, STKF and RSTKF and their results were compared using visual inspection and atlas labels from the Automated Anatomical Labeling (AAL) atlas [45] to the post-surgical resection as seen in the patient's MRI. 


\section{Results}

\subsection{Source imaging of simulated data}

The left frontal source was localized by LORETA, STKF and RSTKF(7) in the white matter near the left frontal lobe. The source in the left putamen was localized in the white matter by LORETA and STKF and in the left putamen by $\operatorname{RSTKF}(7)$. The source in the thalamus was localized in the left putamen and left pallidum by LORETA, in the left putamen by STKF, and in the left and right thalami by $\operatorname{RSTKF}(7)$. The distances between the simulated and estimated source maxima are listed in Table 1. and the source imaging results of these analyses can be seen in Fig. 5.

[Table 1 about here.]

[Figure 5 about here.]

Furthermore regarding EEG source imaging of the data from the thalamic source via RSTKF, the source was localized in both thalami in all cases and the maxima of the source imaging results were localized in the left thalamus in all cases except for $\operatorname{RSTKF}(5)$, which localized the source maximum in the right thalamus. Additionally, the source localization result of the STKF was present as ghost activity except in the case of RSTKF(6) and RSTKF(7), which showed only thalamic activity. These source imaging results can be seen in Fig. 5

\subsection{Source imaging of single-trial spikes from a presurgical patient of epilepsy}

The SNR values of the five selected spikes are 7.45, 11.74, 9.33, 13.28, and 6.26, respectively [28]. The dominant electrodes for the first and fifth spikes were F7 and AF7. For the second, third, and fourth spikes the dominant electrodes were T9, T7, and T9, respectively. At spike onset, LORETA localized the source in the right calcarine, white matter, middle frontal region, intersection of superior and middle temporal region, and in the left cerebellum, for the five spikes, respectively. STKF produced localizations for the five spikes in the white matter, middle temporal region, intersection of left insula and inferior triangular frontal region, white matter, and intersection of superior and middle left temporal regions. RSTKF localized the sources in the left 
amygdalo-hippocampal complex, left hippocampus, left amygdalo-hippocampal complex, left amygdalo-hippocampal complex, and left hippocampus, for the five spikes, respectively. The source reconstruction results at spike onset are depicted in Fig. 5a, in addition to the post-operative MRI. The anatomical labels of the source maxima are listed in Table 2 .

[Table 2 about here.]

At the spike peak, LORETA localized the source in left amygdalo-hippocampal complex, left middle temporal region, white matter, left middle temporal region, and in the left amygdalo-hippocampal complex, for the five spikes, respectively. STKF produced localizations for the first two spikes in the left amygdalo-hippocampal complex, and in intersection of superior and middle left temporal regions; the sources for the third, fourth and fifth spikes were all localized in the white matter. RSTKF localized the sources in the left amygdalo-hippocampal complex for all five spikes. The source reconstruction results at the spike peak are depicted in Fig. 5b, in addition to the post-operative MRI. The anatomical labels of the source maxima are listed in Table 3.

[Figure 6 about here.]

[Table 3 about here.]

\section{Discussion}

\subsection{Achievements}

In this paper, a novel method, the RSTKF, was introduced which generalizes spatiotemporal Kalman filtering by assuming spatially heterogeneous model parameters, thus allowing for the modeling of multiple regional dynamic processes in the brain. RSTKF(7) was tested on simulated EEG data from the frontal lobe, putamen, and thalamus. Larger localization errors from LORETA and STKF were observed as the depth of the sources was increased. The localization of deep sources in the putamen and thalamus was possible via $\operatorname{RSTKF}(7)$. For these two regions, $\operatorname{RSTKF}(7)$ showed smaller 
localization errors than those of LORETA and STKF. Additionally, the anatomical labels of the resulting source maxima from $\operatorname{RSTKF}(7)$ matched those of the simulated sources. The estimated sources via $\operatorname{RSTKF}(7)$ had a better spatial resolution than those of LORETA and STKF, and no problems such as slow convergence of parameter estimates or strange and unexpected parameter values were observed in parameter estimation. In addition to that, the regional definition was changed from two to seven regions to test the accuracy of the localization of the thalamic source. In all cases, the thalamic source was localized and, except for the case of 6 and 7 regions, residuals from the STKF results were observed as ghost activity. We believe that regional definitions with a small number of regions was still to close to the homogeneous model used for STKF. Thus, the ghost sources disappeared when a larger number of regions, 6 or 7 , were used. For the simulated source in the left thalamus, the maximum of the thalamic source was localized in the left hemisphere in all cases except for $\operatorname{RSTKF}(5)$, which showed the maximum of the source in the right hemisphere. The lateralization of the same source by LORETA was correct but the localization was wrong.

Additionally, RSTKF was tested using non-averaged epileptic spikes from a presurgical epilepsy patient with a small lesion in the amygdalo-hippocampal complex. RSTKF(7), unlike LORETA or STKF, consistently and accurately localized the sources for all five spikes at the spikes' onsets and peaks. Furthermore, the spatial resolution of RSTKF(7) was better than that of LORETA or STKF. The parameter estimation process was successful for all spikes. The lateralization of the source was also correct for all spikes.

\subsection{Alternatives and comparisons}

One of the approaches for the localization of subcortical sources is through the use of special forward models. The solution of the EEG inverse problem is not possible without the solution of the EEG forward problem. Forward modeling errors may prevent the localization of deep brain sources. The inclusion of information about the brain dynamics and connectivity in dynamic lead field mapping approach [46] improved the sensitivity of MEG and EEG to deeper cortical sources. Another important issue that affects the modeling of deeper brain structures relates to the definition of the source space as a volumetric grid or a surface mesh, and the grid spacing. Another issue is the 
decision about the inclusion or removal of the cerebellum, brainstem and the subcortical structures in the source space. In this context the work of Attal et al. [5-7] has to be mentioned who suggested the "deep brain activity (DBA) realistic forward model". Using this forward model, the localization of subcortical structures might become feasible with standard inverse methods [7].

Another approach for the localization of subcortical sources is through the use of beamformers. Among the standard source reconstruction methods, the family of time-or frequency-domain beamformers such as linearly-constrained minimum variance (LCMV) and dynamic imaging of coherent sources (DICS) [47, 48] beamformers performed well in the reconstruction of deep brain activity [49,-54]. One problem of beamformer-based analyses is the need for estimation of the covariance or crossspectral density matrix. In order to obtain an estimate of good quality, longer data segments or a large number of trials may be needed.

Another approach for the localization of deep sources is to use source reconstruction to project the surface EEG to the source space and then to perform region-ofinterest, atlas-informed or whole-brain connectivity analysis to uncover deep brain sources. This approach, however, depends on the choice of the source reconstruction and connectivity analysis methods and is also affected by the number of electrodes and the quality of the head model [55, 56]. An alternative to the former approach is given by dynamical causal modeling (DCM) [6, 57, 58] which is a hypothesis-based connectivity analysis approach based on physiologically-informed dynamical models. The application of this method, however, requires information about source locations, which can only be obtained by accurate source reconstruction.

Compared to source imaging approaches, independent component analysis (ICA) is solving a different task, since the former maps into physical space, while the latter maps into some unknown space of independent signals. However, it is possible to apply source imaging to data preprocessed by ICA, rather than raw data. Something similar is done when the data are preprocessed by principal component analysis (PCA), for the purpose of dimensionality reduction and suppression of redundant information. PCA produces uncorrelated components while ICA results in independent components. They are, however, similar to some degree, with PCA forming the first 
part of most ICA algorithms. PCA and ICA are usually used in the preprocessing step for artifact suppression as well as extraction of desired signal components. PCA and most ICA algorithms, however, do not access or utilize the temporal information in the signals. State-space modeling and Kalman filtering were used in [59] to perform source separation and utilize the dynamical information of the EEG signal.

The assumption that cortical sources are characterized by spatial sparsity allowed for the localization of both cortical and subcortical sources via a hierarchical subspacepursuit algorithm [60]. This approach produced promising results in the localization of activity from the thalamus and the brainstem.

Compared to the above-mentioned approaches, RSTKF offers several advantages. Unlike the methods that rely on dynamic lead field mapping or the DBA forward model, RSTKF uses a standard forward model based on a 3D volumetric grid. LORETA and STKF may suffer from the choice of the 3D grid or the definition of the Laplacian matrix [27, 28]. The RSTKF, however, does not seem to be affected by these factors. Unlike the beamformer approaches, it does not require the estimation of the covariance or cross-spectral density matrices and long EEG segments for the analysis. Additionally, the RSTKF does not need to rely on the accuracy of another inverse method, which may critically depend on the number of electrodes or the signal-to-noise ratio in the data, as in the case of DCM or any other connectivity analysis approach. Compared to PCA or ICA, RSTKF does not risk losing information by removing components that include a mixture of desired and undesired signals or need a subjective choice regarding the choice of which components to suppress. RSTKF can, however, be applied to EEG signals that were preprocessed by PCA or ICA. Finally, unlike sparse approaches, RSTKF does not assume spatial sparsity to localize deep brain activity. This is important for the localization of extended sources in the brain without distortion or partition of these sources.

Another advantage of the RSTKF is given by the fact that it represents a dynamical inverse solution, and as such it is able to produce accurate results with less than 64 EEG electrodes. As has been shown above in the results, such small numbers of electrodes do not lead to any loss of spatial resolution. The traditional optimization scheme in RSTKF was successful in assigning the largest dynamical noise variance to 
the expected area in both simulated and clinical EEG data. The lateralization of the source was also mostly accurate, despite the fact that the definition of the brain regions did not differentiate between left and right structures. In addition to that, all results were obtained from non-averaged single spikes with low SNR levels. No averaged spike was needed by RSTKF to obtain accurate and consistent localization. The localization of spike onset in non-averaged spikes is difficult due to the low SNR level, and RSTKF has been found to be robust against this effect. The number of electrodes and the non-averaged analysis may be relevant for practical application of RSTKF, since at many clinics and research centers EEG recordings are performed with 40 electrodes or fewer, and some recordings do not show a sufficient number of spikes to obtain a reliable averaged spike for source reconstruction.

Generally, it was shown in [18] that the use of a dynamical inverse solution with non-vanishing neighbor interactions in the source space, such as the STKF or RSTKF, may make the subspace of silent sources accessible for source reconstruction. This represents an advantage of STKF and RSTKF over static methods. In the ideal case, all state-space dynamical parameters should be allowed to assume individual values for each grid point or, at least, for each region. Concerns about the feasibility of parameter estimation, however, limit this possibility and lead to assumptions of spatial homogeneity, in the case of STKF, or the definition of a few regions in the brain, in the case of RSTKF. In [32, 61] an alternative approach for varying the dynamical noise variance of the spatiotemporal Kalman filter in space (and time) based on a statespace generalized autoregressive conditional heteroscedasticity (GARCH) model was suggested; however, for the high-dimensional EEG inverse problem, this model still suffers from numerical problems. Additionally, it was not tested for the case of source reconstruction of activity from subcortical sources. Currently, the RSTKF is the only stable generalization of STKF that showed, compared to LORETA and STKF, better spatial resolution, accuracy and the ability to detect deep brain sources.

\subsection{Summary, open questions, and further work}

The development of RSTKF was motivated by the aims of achieving better spatial accuracy and improving the localization of subcortical sources. In the field of epilep- 
tology, malformations in, e.g., the mesial temporal and mesial frontal brain regions can cause epilepsy, and these deep sources need to be accurately localized in presurgical evaluation for successful treatment and seizure freedom [62]. Additionally, the thalamus plays an important role in the initiation and propagation of focal and generalized epilepsies [63]. For these reasons, the development of algorithms for localization of subcortical brain structures from surface EEG is important.

This paper presented only a proof-of-principle study. The analyses presented here were performed using a standard realistically-shaped 3-compartment BEM head model; in future work we intend to take the individual anatomy into account. Furthermore, we intend to validate the new method by using a larger number of datasets, e.g., 5-10 per subject, and a larger number of subjects. Regarding simulations, it may not be feasible to study the error variability of RSTKF via simulations with hundreds of repetitions due to prohibitive computational times. As a compromise, a future study with 20-30 repetitions will be performed. These simulations will be performed with more realistic SNR values (5-35 dB) and more sophisticated dynamical models to avoid inverse crimes. The repetitions would also allow us to plot the distribution of source location results.

We limited the number of regions in this paper to seven. The optimal number of regions could be obtained via ML optimization, by introducing successively more regions, and refitting the model. Then the minimum of AIC would show the optimal number of regions. But this is a simplified picture. When starting with a large number of initial regions, there is a combinatorial multitude of possible sets of larger regions, obtained by merging subsets of smaller regions. For all these sets of regions optimal models would have to be fitted, and their AIC compared. This would be infeasible. In practical work, even fitting a model for one choice of regions is time consuming, and this limits the number of regions that can be employed. The choice of regions to be included in the model may be guided by prior knowledge regarding possibly relevant source locations; but clearly such choice will always introduce a certain subjective bias into the modeling.

The development of the RSTKF method requires the definition of more regions with their own dynamical noise parameters, in order to better reflect the anatomical 
or functional modularity of the brain. Anatomical atlases, such as the AAL atlas [45] that include subcortical areas, contain up to 116 regions. In this paper we concentrated on adding more subcortical structures in the choice of regions. In the future, the subdivision of the cortical areas into lobes or even gyral and sulcar structures will be performed to improve the accuracy of RSTKF for cortical sources. Additionally, the choice of the number of regions needs to be a compromise between a small number of regions, which may be too close to the STKF, and a large number, which may cause larger computational times, identifiability problems and local minima for the optimization algorithms. A larger number of regions may be feasible through a "restricted optimization" approach in which subgroups of parameters are optimized.

In this study we chose to make the MA parameters spatially heterogeneous. The idea to have space-dependent AR parameters, however, is not unreasonable. AR parameters determine the oscillation frequency, so if different parts of the brain generate oscillations with different frequencies, this may be reflected by different AR parameters. This would be a different type of RSTKF model, for which variance, or MA parameters, would be independent of region.

Regarding the choice of the source gird, we intend to use $5 \mathrm{~mm}$ grid spacing in order to represent the small or longitudinal subcortical structures with enough grid points. In addition to the grid choice, we used only the classical definition of the Laplacian matrix in this study since it results in a non-singular Laplacian matrix. The use of this Laplacian matrix, however, suppresses the activity at the borders of the grid, since it always assumes the presence of six neighbors to each grid point, which is not true for grid points at the borders of the grid [28, 64]. The use of the six neighbors in the $x$, $y$, and $z$ directions was also chosen because it is common in these kinds of studies. In the future, the neighbors in the diagonal directions can be included in the Laplacian matrix. Additionally, we intend to test the modified definition of the Laplacian matrix from [64] for RSTKF analyses. AIC comparison may be used in the future to compare different definitions of the Laplacian matrix.

The the current study was performed only for single brain sources. We would like to emphasize, however, that the activity of the whole brain is estimated by each of the the three algorithms without any specification of the number of sources prior to source 
imaging. The use of single sources was only for validation and not as a parameter for the source imaging algorithm. We only used the Euclidian distance as a performance measure without any averaging or cross validation. A possible compromise would be to have distribution of source location results.. We will use the earth mover's distance (EMD) [65] in the future to compare the spatial distributions of the simulated and reconstructed current densities. In a future study we also intend to investigate the applications in epileptology or cognitive neuroscience in which simultaneous activations of several subcortical sources, or of cortical and subcortical sources. In these cases a masking effect may be observed [12] and additional preprocessing steps involving filtering or ICA may be needed to disentangle the effects of different sources prior to source imaging. Additionally, these analyses will show how long-range connectivities are affected by the nearest-neighbor state-space representation, since this is interesting for investigations of propagated activity in epileptology.

Since the whole brain activity is estimated at the same time and not sequentially, there is a potential for reduction of the computational time. The computational time is linearly proportional to the number of voxels, time points and optimization steps. It is, however, non-linearly proportional to the number of electrodes and that is why we employ dimensionality reduction approaches when we deal with high-density EEG data. Model reduction approaches, e.g. based on singular value decomposition (SVD), may be very helpful in improving the computational speed of the RSTKF [31]

The next step will be to use individual head models and individual atlases of brain regions. The localization of subcortical sources may become easier through the reduction in head modeling errors [66]. This could be achieved by using detailed state-ofthe-art 6-compartment finite element (FE) head models, which model the anisotropic white matter, gray matter, cerebrospinal fluid (CSF), three layer skull, and skin [4, 67]. Finally the important cases of simultaneous subcortical activations or simultaneous cortical and subcortical activations still need to be investigated using simulated and clinical EEG data. The validation of the RSTKF approach may be best performed by analyzing simultaneous recordings from surface EEG and from depth electrodes, or by analyzing surface EEG recordings of activity from deep brain stimulation (DBS) of the thalamus or the subthalamic nucleus (STN) [68]. 


\section{Conclusion}

In summary, RSTKF is a dynamical source imaging approach which outperformed both LORETA and STKF in both accuracy and spatial resolution based on the data analysis that was performed in this paper. The new method has shown promising results in the case of deep brain sources from EEG recordings with fewer than 64 electrodes, and without the need for averaged spikes. In the future, validation models with DBS or simultaneous EEG and depth recordings will be used to validate the RSTKF.

\section{Acknowledgements}

Laith Hamid, Natia Japaridze and Andreas Galka were funded by the D3 subproject of the Collaborative Research Center CRC855 "Magnetoelectric Composites â biomagnetic interfaces of the future" of the German Science Society (DFG). Laith Hamid, Nawar Habboush and Andreas Galka are funded by the B3 subproject of the Collaborative Research Center CRC 1261 âMagnetoelectric Sensors: From Composite Materials to Biomagnetic Diagnosticsââ of the DFG, http://www.sfb1261.de/index.php/en/. Laith Hamid and Natia Japaridze were also funded by the European Union's Seventh Framework Programme for research, technological development and demonstration through the project DESIRE "Development \& Epilepsy" (Grant Agreement no: 602531), WP2 \& WP4, http://epilepsydesireproject.eu/. Ümit Aydin and Carsten H Wolters were financed by DFG projects WO1425/2-1,7-1.

The authors would like to thank Dr. med. Lydia Elshoff and Dr. med. Gert Wiegand from the Department of Neuropediatrics, University of Kiel, Germany, for providing the clinical EEG data. We are also grateful for the help from our students and colleagues, especially Prof. Dr.-Ing. Gerhard Schmidt from the Digital Signal Processing and System Theory Group at the University of Kiel, Germany. Furthermore, the authors acknowledge the work of Juan Pablo Neira on the visualization of the EEG source imaging results. 


\section{References}

[1] F. L. da Silva, EEG and MEG: relevance to neuroscience, Neuron 80 (2013) $1112-1128$.

[2] C. M. Michel, M. M. Murray, Towards the utilization of EEG as a brain imaging tool, Neuroimage 61 (2012) 371-385.

[3] F. H. L. da Silva, The impact of EEG/MEG signal processing and modeling in the diagnostic and management of epilepsy, IEEE Reviews in Biomedical Engineering 1 (2008) 143-156.

[4] Ü. Aydin, J. Vorwerk, P. Küpper, M. Heers, H. Kugel, A. Galka, L. Hamid, J. Wellmer, C. Kellinghaus, S. Rampp, et al., Combining EEG and MEG for the reconstruction of epileptic activity using a calibrated realistic volume conductor model, PLoS One 9 (2014).

[5] Y. Attal, M. Bhattacharjee, J. Yelnik, B. Cottereau, J. Lefèvre, Y. Okada, E. Bardinet, M. Chupin, S. Baillet, Modeling and detecting deep brain activity with MEG \& EEG, in: $200729^{\text {th }}$ Annual International Conference of the IEEE Engineering in Medicine and Biology Society, IEEE, 2007, pp. 4937-4940.

[6] Y. Attal, B. Maess, A. Friederici, O. David, Head models and dynamic causal modeling of subcortical activity using magnetoencephalographic/electroencephalographic data, Reviews in the Neurosciences 23 (2012) 85-95.

[7] Y. Attal, D. Schwartz, Assessment of subcortical source localization using deep brain activity imaging model with minimum norm operators: a MEG study, PLoS One 8 (2013).

[8] D. Muzumdar, M. Patil, A. Goel, S. Ravat, N. Sawant, U. Shah, Mesial temporal lobe epilepsy-an overview of surgical techniques, International Journal of Surgery 36 (2016) 411-419. 
[9] S. Baillet, J. C. Mosher, R. M. Leahy, Electromagnetic brain mapping, IEEE Signal Processing Magazine 18 (2001) 14-30.

[10] R. Grech, T. Cassar, J. Muscat, K. P. Camilleri, S. G. Fabri, M. Zervakis, P. Xanthopoulos, V. Sakkalis, B. Vanrumste, Review on solving the inverse problem in EEG source analysis, Journal of Neuroengineering and Rehabilitation 5 (2008) 25.

[11] M. A. Jatoi, N. Kamel, A. S. Malik, I. Faye, T. Begum, A survey of methods used for source localization using EEG signals, Biomedical Signal Processing and Control 11 (2014) 42-52.

[12] F. Lucka, S. Pursiainen, M. Burger, C. H. Wolters, Hierarchical Bayesian inference for the EEG inverse problem using realistic FE head models: depth localization and source separation for focal primary currents, NeuroImage 61 (2012) $1364-1382$.

[13] X. Lei, T. Wu, P. Valdes-Sosa, Incorporating priors for EEG source imaging and connectivity analysis, Frontiers in Neuroscience 9 (2015) 284.

[14] R. P. Marqui, C. M. Michel, D. Lehmann, Low-resolution electromagnetic tomography-a new method for localizing electrical activity in the brain, International Journal of Psychophysiology 18 (1994) 49-65.

[15] R. D. Pascual-Marqui, et al., Standardized low-resolution brain electromagnetic tomography (sLORETA): technical details, Methods and Findings in Experimental and Clinical Pharmacology 24 (2002) 5-12.

[16] M. Dannhauer, E. Lämmel, C. H. Wolters, T. R. Knösche, Spatio-temporal regularization in linear distributed source reconstruction from EEG/MEG: a critical evaluation, Brain Topography 26 (2013) 229-246.

[17] U. Schmitt, A. K. Louis, C. Wolters, M. Vauhkonen, Efficient algorithms for the regularization of dynamic inverse problems: II. applications, Inverse Problems 18 (2002) 659. 
[18] A. Galka, O. Yamashita, T. Ozaki, R. Biscay, P. Valdés-Sosa, A solution to the dynamical inverse problem of EEG generation using spatiotemporal Kalman filtering, NeuroImage 23 (2004) 435-453.

[19] T. Ozaki, Time series modeling of neuroscience data, CRC Press, 2012.

[20] R. Kalman, A new approach to linear filtering and prediction problems, Transactions of the ASME-Journal of Basic Engineering 82 (1960) 35-45.

[21] D. Simon, Optimal state estimation: Kalman, H infinity, and nonlinear approaches, John Wiley \& Sons, 2006.

[22] S. Lakshmivarahan, D. J. Stensrud, Ensemble Kalman filter, IEEE Control Systems Magazine 29 (2009) 34-46.

[23] P. Brasseur, Ocean data assimilation using sequential methods based on the Kalman filter, in: Ocean Weather Forecasting, Springer, 2006, pp. 271-316.

[24] A. Heemink, A. Segers, Modeling and prediction of environmental data in space and time using Kalman filtering, Stochastic Environmental Research and Risk Assessment 16 (2002) 225-240.

[25] G. Nakamura, R. Potthast, Inverse modeling, IOP Publishing, 2015.

[26] S. J. Schiff, Neural control engineering: the emerging intersection between control theory and neuroscience, MIT Press, 2012.

[27] L. Hamid, M. Sarabi, N. Japaridze, G. Wiegand, U. Heute, U. Stephani, A. Galka, M. Siniatchkin, The performance of the spatiotemporal Kalman filter and LORETA in seizure onset localization, in: $201537^{\text {th }}$ Annual International Conference of the IEEE Engineering in Medicine and Biology Society (EMBC), IEEE, 2015, pp. 2741-2744.

[28] N. Habboush, L. Hamid, N. Japaridze, G. Wiegand, U. Heute, U. Stephani, A. Galka, M. Siniatchkin, The choice of the source space and the laplacian matrix in LORETA and the spatio-temporal Kalman filter EEG inverse methods, in: 
$201537^{\text {th }}$ Annual International Conference of the IEEE Engineering in Medicine and Biology Society (EMBC), IEEE, 2015, pp. 2745-2749.

[29] L. Hamid, Ü. Aydin, C. Wolters, U. Stephani, M. Siniatchkin, A. Galka, MEGEEG fusion by Kalman filtering within a source analysis framework, in: 2013 $35^{\text {th }}$ Annual International Conference of the IEEE Engineering in Medicine and Biology Society (EMBC), IEEE, 2013, pp. 4819-4822.

[30] L. Hamid, A. Al Farawn, I. Merlet, N. Japaridze, U. Heute, U. Stephani, A. Galka, F. Wendling, M. Siniatchkin, Source reconstruction via the spatiotemporal Kalman filter and LORETA from EEG time series with 32 or fewer electrodes, in: $201739^{\text {th }}$ Annual International Conference of the IEEE Engineering in Medicine and Biology Society (EMBC), IEEE, 2017, pp. 2218-2222.

[31] L. Hamid, A. Al Farawn, I. Merlet, N. Japaridze, U. Heute, U. Stephani, A. Galka, F. Wendling, M. Siniatchkin, Spatial projection as a preprocessing step for EEG source reconstruction using spatiotemporal Kalman filtering, in: $201739^{\text {th }}$ Annual International Conference of the IEEE Engineering in Medicine and Biology Society (EMBC), IEEE, 2017, pp. 2213-2217.

[32] A. Galka, T. Ozaki, H. Muhle, U. Stephani, M. Siniatchkin, A data-driven model of the generation of human EEG based on a spatially distributed stochastic wave equation, Cognitive Neurodynamics 2 (2008) 101.

[33] O. Yamashita, A. Galka, T. Ozaki, R. Biscay, P. Valdes-Sosa, Recursive penalized least squares solution for dynamical inverse problems of EEG generation, Human Brain Mapping 21 (2004) 221-235.

[34] R. Oostenveld, P. Fries, E. Maris, J.-M. Schoffelen, Fieldtrip: open source software for advanced analysis of MEG, EEG, and invasive electrophysiological data, Computational Intelligence and Neuroscience 2011 (2011).

[35] T. R. Knösche, M. Gräser, A. Anwander, Prior knowledge on cortex organization in the reconstruction of source current densities from EEG, Neuroimage 67 (2013) 7-24. 
[36] H. Wold, A study in the analysis of stationary time series, Ph.D. thesis, Almqvist \& Wiksell, 1938.

[37] A. Galka, T. Ozaki, J. B. Bayard, O. Yamashita, Whitening as a tool for estimating mutual information in spatiotemporal data sets, Journal of Statistical Physics 124 (2006) 1275-1315.

[38] M. J. Barton, P. A. Robinson, S. Kumar, A. Galka, H. F. Durrant-Whyte, J. Guivant, T. Ozaki, Evaluating the performance of Kalman-filter-based EEG source localization, IEEE Transactions on Biomedical Engineering 56 (2008) 122-136.

[39] H. Akaike, Information theory as an extension of the maximum likelihood principle., in: B. N. Petrov, F. Csaki (Eds.), Second International Symposium on Information Theory. Akademiai Kiado, Budapest, pp. 276-281, 1973.

[40] A. C. Harvey, Forecasting, structural time series models and the Kalman filter, Cambridge university press, 2008.

[41] T. Moberget, R. B. Ivry, Cerebellar contributions to motor control and language comprehension: searching for common computational principles, Annals of the New York Academy of Sciences 1369 (2016) 154.

[42] J. Engel Jr, A proposed diagnostic scheme for people with epileptic seizures and with epilepsy: report of the ilae task force on classification and terminology, Epilepsia 42 (2001) 796-803.

[43] J. Engel Jr, Outcome with respect to epileptic seizures., Surgical Treatment of the Epilepsies (1993) 609-621.

[44] V. P. Oikonomou, A. T. Tzallas, D. I. Fotiadis, A Kalman filter based methodology for EEG spike enhancement, Computer Methods and Programs in Biomedicine 85 (2007) 101-108.

[45] N. Tzourio-Mazoyer, B. Landeau, D. Papathanassiou, F. Crivello, O. Etard, N. Delcroix, B. Mazoyer, M. Joliot, Automated anatomical labeling of activations in SPM using a macroscopic anatomical parcellation of the MNI MRI single-subject brain, Neuroimage 15 (2002) 273-289. 
[46] C. Lamus, M. S. Hamalainen, E. N. Brown, P. L. Purdon, An analysis of how spatiotemporal dynamic models of brain activity could improve MEG/EEG inverse solutions, arXiv preprint arXiv:1511.03726 (2015).

[47] J. Groß, J. Kujala, M. Hämäläinen, L. Timmermann, A. Schnitzler, R. Salmelin, Dynamic imaging of coherent sources: studying neural interactions in the human brain, Proceedings of the National Academy of Sciences 98 (2001) 694-699.

[48] C. Micheli, C. Braun, Enhancing the signal of corticomuscular coherence, Computational and Mathematical Methods in Medicine 2012 (2012).

[49] N. Japaridze, M. Muthuraman, C. Dierck, S. von Spiczak, R. Boor, K. G. Mideksa, R. A. Anwar, G. Deuschl, U. Stephani, M. Siniatchkin, Neuronal networks in epileptic encephalopathies with CSWS, Epilepsia 57 (2016) 1245-1255.

[50] N. Japaridze, M. Muthuraman, F. Moeller, R. Boor, A. R. Anwar, G. Deuschl, U. Stephani, J. Raethjen, M. Siniatchkin, Neuronal networks in West syndrome as revealed by source analysis and renormalized partial directed coherence, Brain Topography 26 (2013) 157-170.

[51] L. Michels, M. Muthuraman, R. Lüchinger, E. Martin, A. R. Anwar, J. Raethjen, D. Brandeis, M. Siniatchkin, Developmental changes of functional and directed resting-state connectivities associated with neuronal oscillations in EEG, Neuroimage 81 (2013) 231-242.

[52] F. Moeller, M. Muthuraman, U. Stephani, G. Deuschl, J. Raethjen, M. Siniatchkin, Representation and propagation of epileptic activity in absences and generalized photoparoxysmal responses, Human Brain Mapping 34 (2013) 18961909.

[53] L. Elshoff, M. Muthuraman, A. R. Anwar, G. Deuschl, U. Stephani, J. Raethjen, M. Siniatchkin, Dynamic imaging of coherent sources reveals different network connectivity underlying the generation and perpetuation of epileptic seizures, PloS One 8 (2013). 
[54] M. Muthuraman, G. Deuschl, J. Raethjen, Essential constraints for detecting deep sources in EEG-application to orthostatic tremor, in: $20114^{\text {th }}$ International Congress on Image and Signal Processing, volume 5, IEEE, 2011, pp. 2729-2732.

[55] J.-M. Schoffelen, J. Gross, Source connectivity analysis with MEG and EEG, Human Brain Mapping 30 (2009) 1857-1865.

[56] A. M. Bastos, J.-M. Schoffelen, A tutorial review of functional connectivity analysis methods and their interpretational pitfalls, Frontiers in Systems Neuroscience 9 (2016) 175.

[57] O. David, S. J. Kiebel, L. M. Harrison, J. Mattout, J. M. Kilner, K. J. Friston, Dynamic causal modeling of evoked responses in EEG and MEG, NeuroImage 30 (2006) 1255-1272.

[58] O. David, B. Maess, K. Eckstein, A. D. Friederici, Dynamic causal modeling of subcortical connectivity of language, Journal of Neuroscience 31 (2011) 27122717.

[59] A. Galka, K. F. K. Wong, T. Ozaki, H. Muhle, U. Stephani, M. Siniatchkin, Decomposition of neurological multivariate time series by state space modelling, Bulletin of Mathematical Biology 73 (2011) 285-324.

[60] P. Krishnaswamy, G. Obregon-Henao, J. Ahveninen, S. Khan, B. Babadi, J. E. Iglesias, M. S. Hämäläinen, P. L. Purdon, Sparsity enables estimation of both subcortical and cortical activity from MEG and EEG, Proceedings of the National Academy of Sciences 114 (2017) E10465-E10474.

[61] A. Galka, O. Yamashita, T. Ozaki, GARCH modelling of covariance in dynamical estimation of inverse solutions, Physics Letters A 333 (2004) 261-268.

[62] C. P. Panayiotopoulos, The epilepsies: seizures, syndromes and management, Bladon Medical Publishing, Oxfordshire (UK), 2005.

[63] A. Cukiert, K. Lehtimäki, Deep brain stimulation targeting in refractory epilepsy, Epilepsia 58 (2017) 80-84. 
[64] R. D. Pascual-Marqui, Review of methods for solving the EEG inverse problem, International Journal of Bioelectromagnetism 1 (1999) 75-86.

[65] Y. Rubner, C. Tomasi, L. J. Guibas, The earth mover's distance as a metric for image retrieval, International Journal of Computer Vision 40 (2000) 99-121.

[66] C. H. Wolters, A. Anwander, X. Tricoche, D. Weinstein, M. A. Koch, R. S. Macleod, Influence of tissue conductivity anisotropy on EEG/MEG field and return current computation in a realistic head model: a simulation and visualization study using high-resolution finite element modeling, NeuroImage 30 (2006) $813-826$.

[67] J. Vorwerk, J.-H. Cho, S. Rampp, H. Hamer, T. R. Knösche, C. H. Wolters, A guideline for head volume conductor modeling in EEG and MEG, NeuroImage 100 (2014) 590-607.

[68] M. Muthuraman, S. Paschen, H. Hellriegel, S. Groppa, G. Deuschl, J. Raethjen, Locating the STN-DBS electrodes and resolving their subsequent networks using coherent source analysis on EEG, in: $201234^{\text {th }}$ Annual International Conference of the IEEE Engineering in Medicine and Biology Society, IEEE, 2012, pp. 39703973. 
817

818

819

820

821

822

823

824

825

826

827

828

829

830

831

832

833

834

835

836

837

838

839

840

841

842

843

844

845

846

847

848

849

850

851

852

853

854

855

\section{List of Figures}

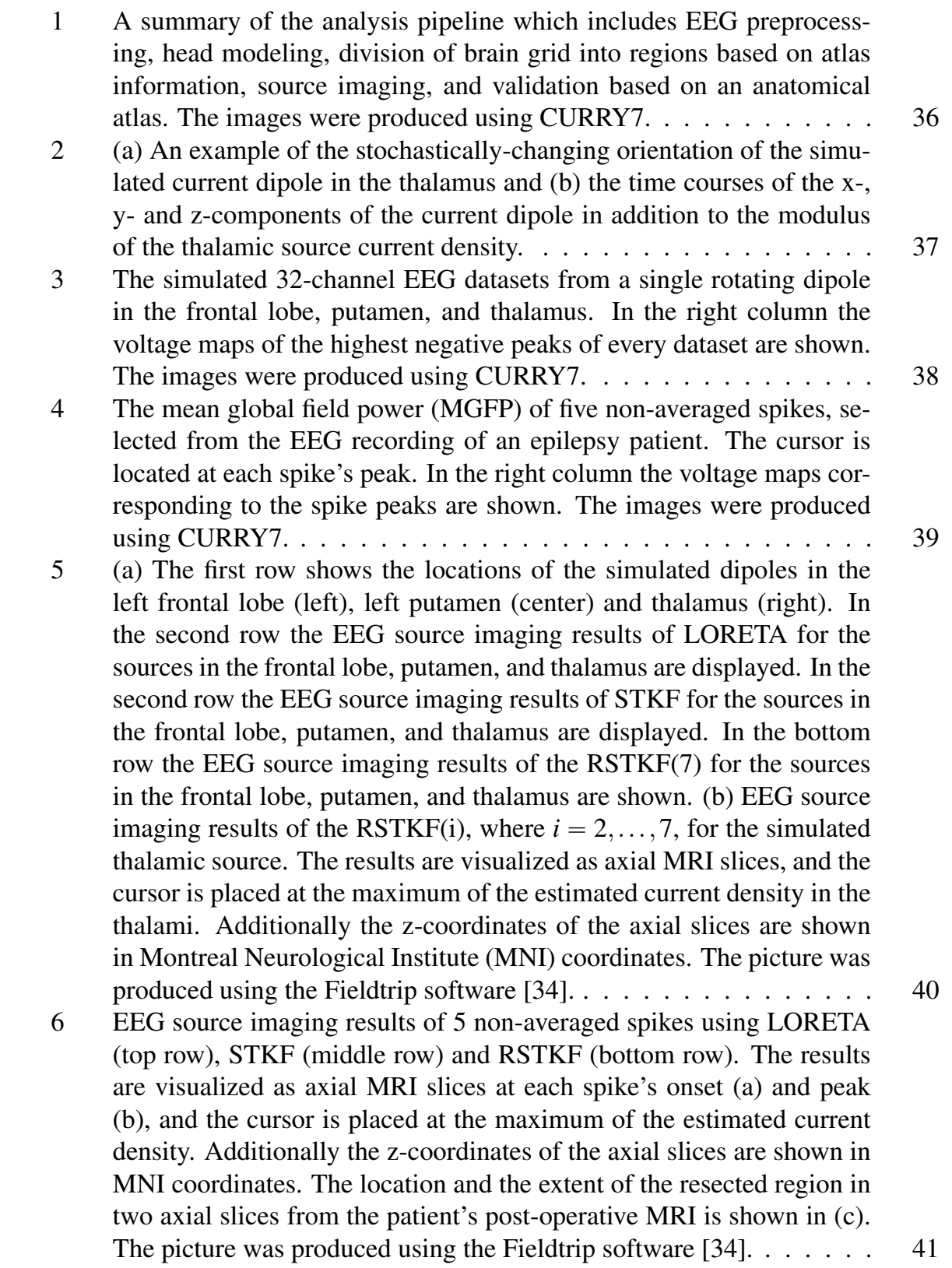




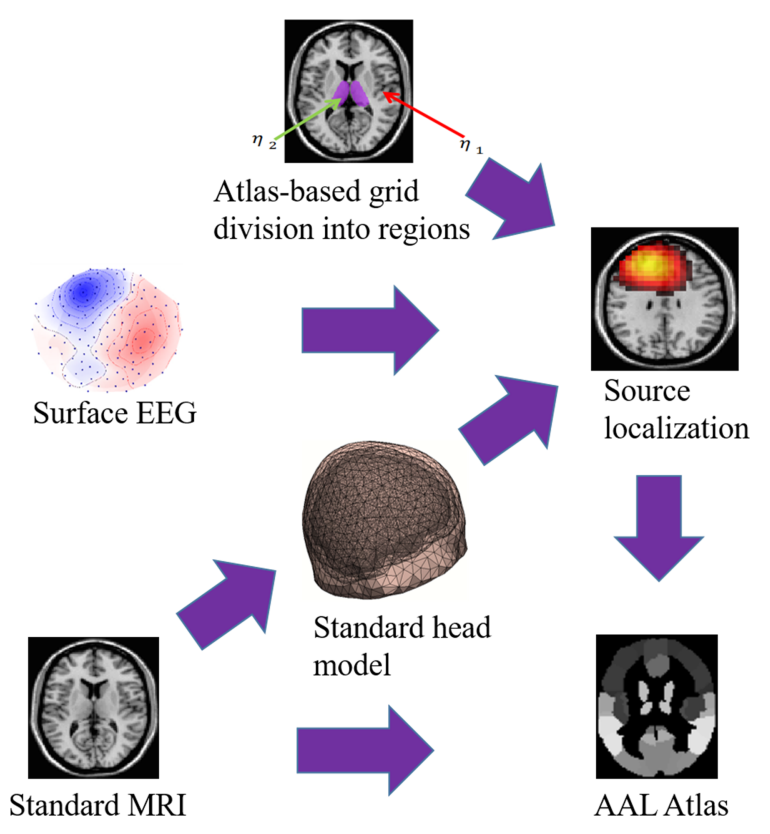

Figure 1: A summary of the analysis pipeline which includes EEG preprocessing, head modeling, division of brain grid into regions based on atlas information, source imaging, and validation based on an anatomical atlas. The images were produced using CURRY7. 
(a)
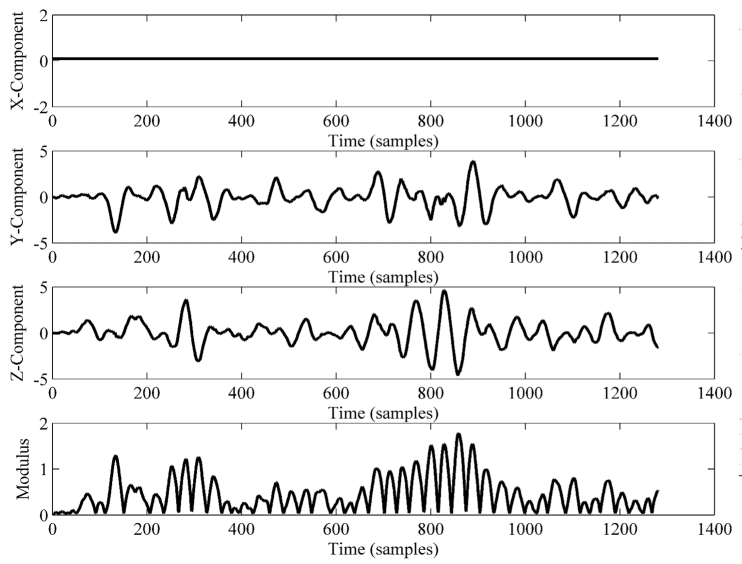

(b)

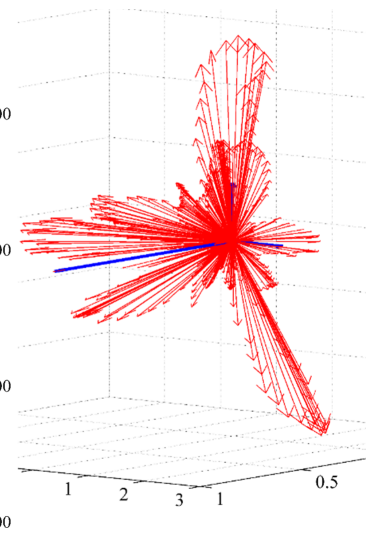

Figure 2: (a) An example of the stochastically-changing orientation of the simulated current dipole in the thalamus and (b) the time courses of the $\mathrm{x}$-, $\mathrm{y}$ - and z-components of the current dipole in addition to the modulus of the thalamic source current density. 


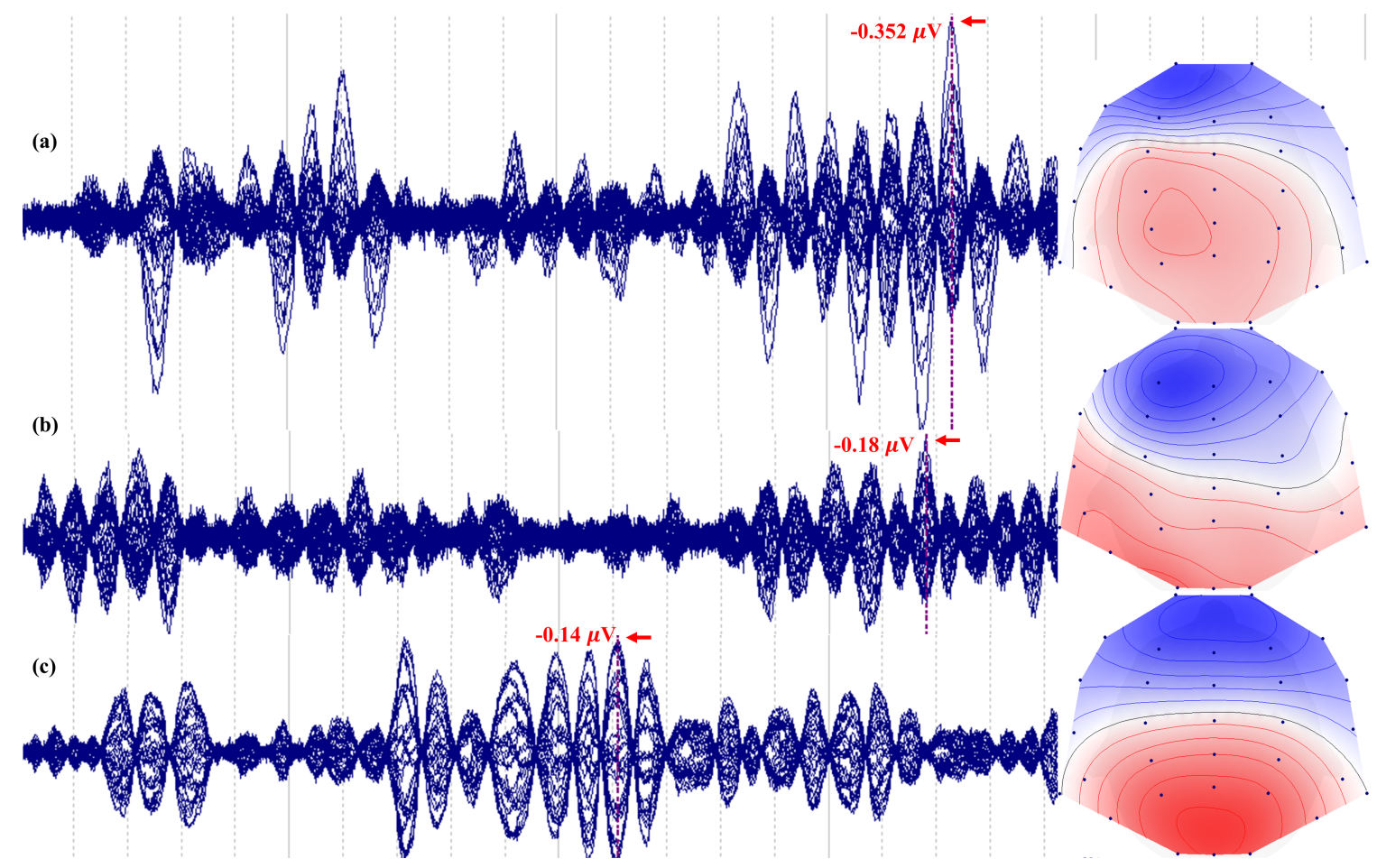

Figure 3: The simulated 32-channel EEG datasets from a single rotating dipole in the frontal lobe, putamen, and thalamus. In the right column the voltage maps of the highest negative peaks of every dataset are shown. The images were produced using CURRY7. 


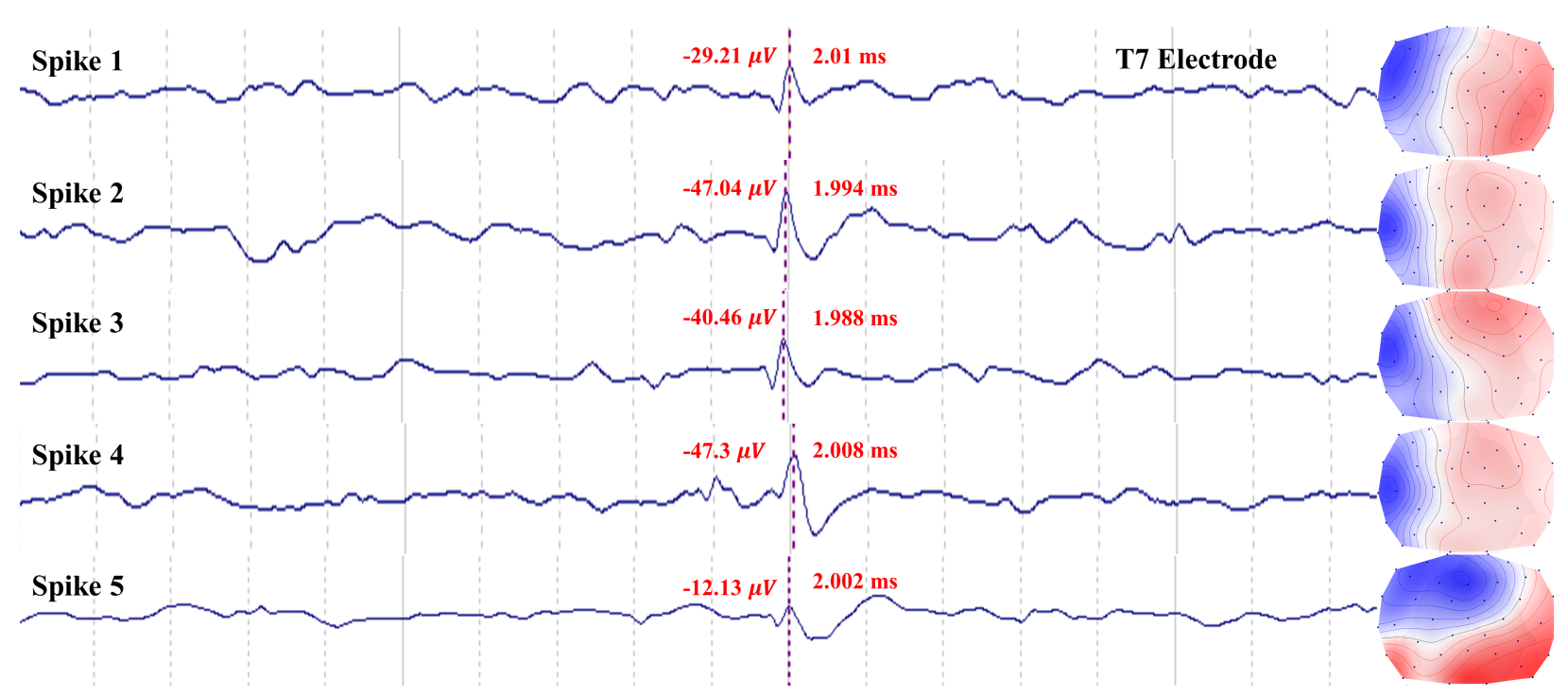

Figure 4: The mean global field power (MGFP) of five non-averaged spikes, selected from the EEG recording of an epilepsy patient. The cursor is located at each spike's peak. In the right column the voltage maps corresponding to the spike peaks are shown. The images were produced using CURRY7. 


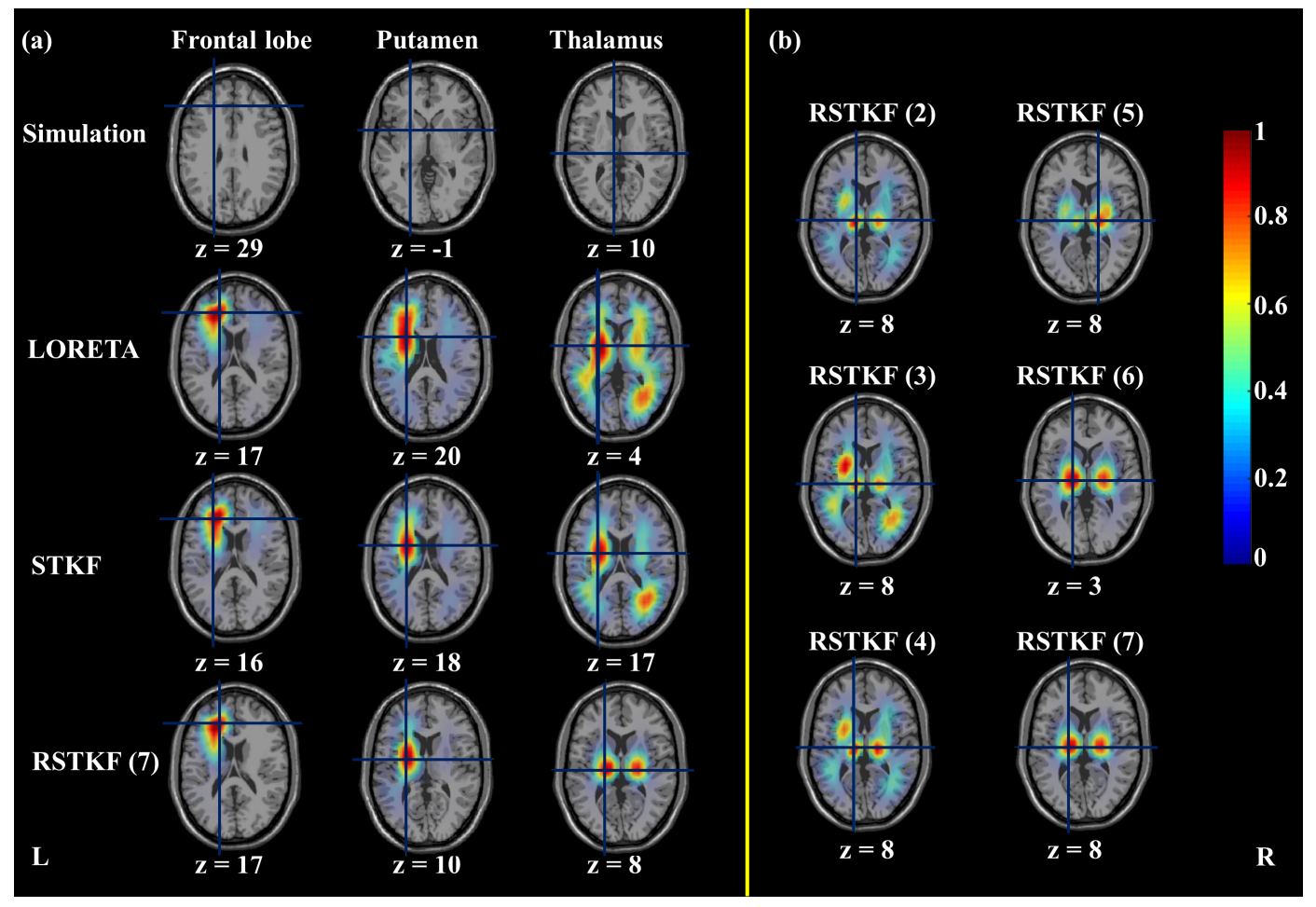

Figure 5: (a) The first row shows the locations of the simulated dipoles in the left frontal lobe (left), left putamen (center) and thalamus (right). In the second row the EEG source imaging results of LORETA for the sources in the frontal lobe, putamen, and thalamus are displayed. In the second row the EEG source imaging results of STKF for the sources in the frontal lobe, putamen, and thalamus are displayed. In the bottom row the EEG source imaging results of the $\operatorname{RSTKF}(7)$ for the sources in the frontal lobe, putamen, and thalamus are shown. (b) EEG source imaging results of the RSTKF(i), where $i=2, \ldots, 7$, for the simulated thalamic source. The results are visualized as axial MRI slices, and the cursor is placed at the maximum of the estimated current density in the thalami. Additionally the z-coordinates of the axial slices are shown in Montreal Neurological Institute (MNI) coordinates. The picture was produced using the Fieldtrip software [34]. 


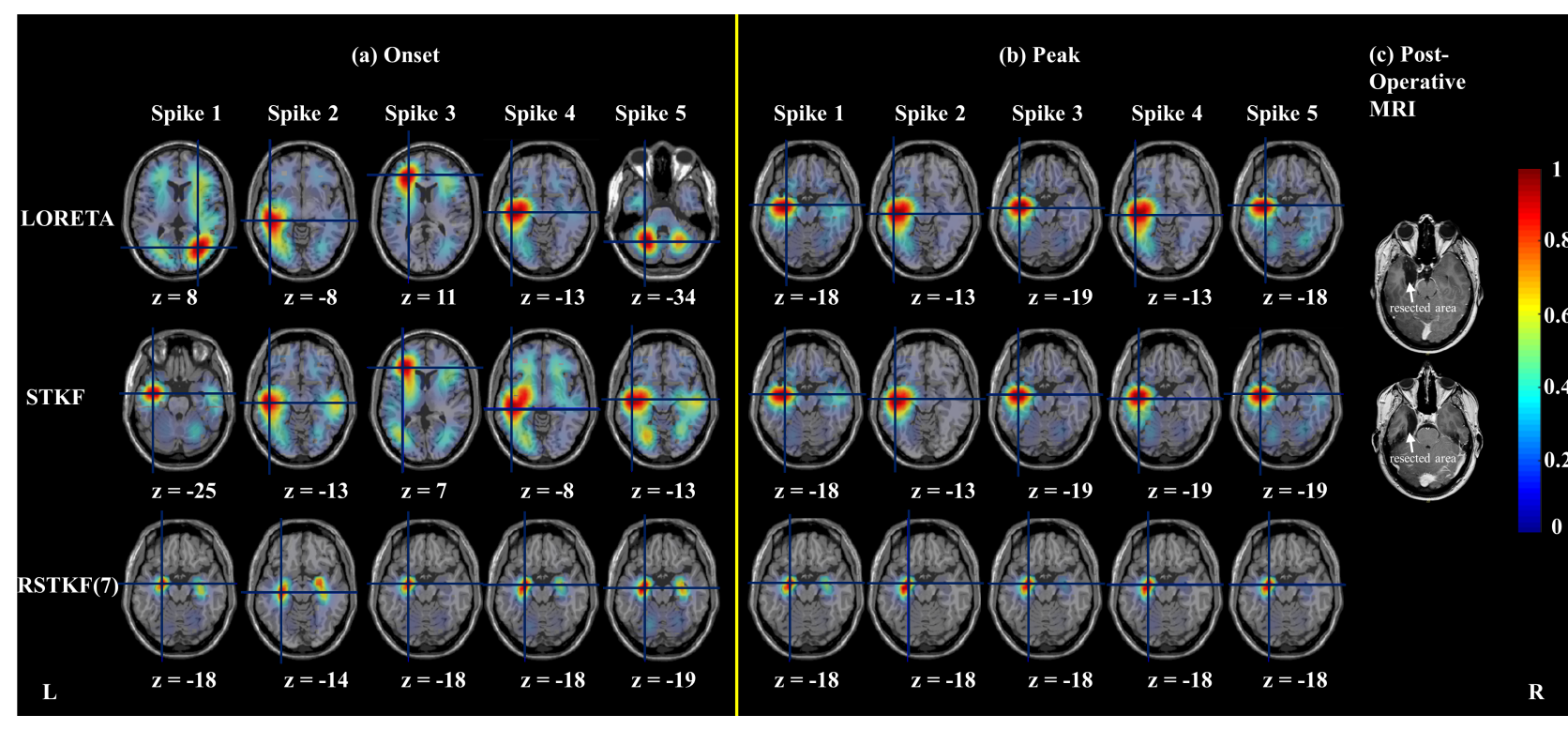

Figure 6: EEG source imaging results of 5 non-averaged spikes using LORETA (top row), STKF (middle row) and RSTKF (bottom row). The results are visualized as axial MRI slices at each spike's onset (a) and peak (b), and the cursor is placed at the maximum of the estimated current density. Additionally the z-coordinates of the axial slices are shown in MNI coordinates. The location and the extent of the resected region in two axial slices from the patient's post-operative MRI is shown in (c). The picture was produced using the Fieldtrip software [34]. 


\section{List of Tables}

\begin{tabular}{|c|c|c|}
\hline & 1 & Euclidean distance (in $\mathrm{mm}$ ) between the simulated and $\mathrm{e}$ \\
\hline & & rent density maxima using LORETA, STKF, and RSTKF as EEG source \\
\hline & & imaging methods for the simulated sources in the frontal lobe, putamen \\
\hline & & 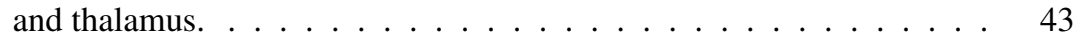 \\
\hline & 2 & EEG source imaging results of five non-averaged spikes selected from \\
\hline & & the EEG recording of an epilepsy patient. The epileptic focus lies in the \\
\hline & & amygdalo-hippocampal area. The results were visualized at the spikes' \\
\hline & & onsets defined as $50 \%$ of the spike's rise time. For the maximum source \\
\hline & & activation, anatomical labels from the AAL atlas are shown. If the max- \\
\hline & & imum of the estimated source activity falls within the resected region, \\
\hline & & the result is concordant, $-\mathrm{C}-$, with the resected region; if the maximum \\
\hline & & does not fall within the resected region, the result is disconcordant, \\
\hline & & -DC-, with the resected region. $\ldots \ldots \ldots \ldots \ldots \ldots$ \\
\hline & 3 & EEG source imaging results of five non-averaged spikes selected from \\
\hline & & the EEG recording of an epilepsy patient. The epileptic focus lies in the \\
\hline & & amygdalo-hippocampal area. The results were visualized at the spikes' \\
\hline & & peaks. For the maximum source activation, anatomical labels from \\
\hline & & the AAL atlas are shown. If the maximum of the estimated source \\
\hline & & activity falls within the resected region, the result is concordant, -C- \\
\hline & & , with the resected region; if the maximum does not fall within the \\
\hline & & resected region, the result is disconcordant, -DC-, with the resected \\
\hline & & 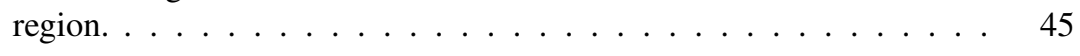 \\
\hline
\end{tabular}


Table 1: Euclidean distance (in $\mathrm{mm}$ ) between the simulated and estimated current density maxima using LORETA, STKF, and RSTKF as EEG source imaging methods for the simulated sources in the frontal lobe, putamen and thalamus.

\begin{tabular}{|c|c|c|c|}
\hline Method & $\begin{array}{l}\text { Euclidean dis- } \\
\text { tance (in } \mathrm{mm}) \\
\text { between the } \\
\text { simulated and } \\
\text { reconstructed }\end{array}$ & $\begin{array}{l}\text { Euclidean dis- } \\
\text { tance (in } \mathrm{mm} \text { ) } \\
\text { between the } \\
\text { simulated and } \\
\text { reconstructed }\end{array}$ & $\begin{array}{l}\text { Euclidean dis- } \\
\text { tance (in mm) } \\
\text { between the } \\
\text { simulated and } \\
\text { reconstructed }\end{array}$ \\
\hline & $\begin{array}{l}\text { sources in the } \\
\text { frontal lobe }\end{array}$ & $\begin{array}{l}\text { sources in the } \\
\text { putamen }\end{array}$ & $\begin{array}{l}\text { sources in the } \\
\text { thalamus }\end{array}$ \\
\hline LORETA & 12.8062 & 20.8327 & 25.7099 \\
\hline STKF & 15.2971 & 20.3224 & 24.5967 \\
\hline RSTKF & 12.8062 & 16.0935 & 11.3578 \\
\hline
\end{tabular}


Table 2: EEG source imaging results of five non-averaged spikes selected from the EEG recording of an epilepsy patient. The epileptic focus lies in the amygdalo-hippocampal area. The results were visualized at the spikes' onsets defined as $50 \%$ of the spike's rise time. For the maximum source activation, anatomical labels from the AAL atlas are shown. If the maximum of the estimated source activity falls within the resected region, the result is concordant, $-\mathrm{C}$-, with the resected region; if the maximum does not fall within the resected region, the result is disconcordant, -DC-, with the resected region.

\begin{tabular}{|c|c|c|c|}
\hline Datasets & $\begin{array}{l}\text { Anatomical labels } \\
\text { of LORETA result }\end{array}$ & $\begin{array}{l}\text { Anatomical labels } \\
\text { of STKF result }\end{array}$ & $\begin{array}{l}\text { Anatomical labels } \\
\text { of RSTKF result }\end{array}$ \\
\hline Spike 1 onset & $\begin{array}{l}\text { Calcarine R } \\
\text {-DC- }\end{array}$ & $\begin{array}{l}\text { Temporal Mid L } \\
\text {-DC- }\end{array}$ & $\begin{array}{l}\text { Amygdala L, Hip- } \\
\text { pocampus L } \\
\text {-C- }\end{array}$ \\
\hline Spike 2 onset & $\begin{array}{l}\mathrm{N} \backslash \mathrm{A} \\
-\mathrm{DC}-\end{array}$ & $\begin{array}{l}\text { Temporal Mid L } \\
\text {-DC- }\end{array}$ & $\begin{array}{l}\text { Hippocampus L } \\
-\mathrm{C}-\end{array}$ \\
\hline Spike 3 onset & $\begin{array}{l}N \backslash A \\
-D C-\end{array}$ & $\begin{array}{l}\text { Frontal Inf Tri L } \\
\text {-DC- }\end{array}$ & $\begin{array}{l}\text { Amygdala L, Hip- } \\
\text { pocampus L } \\
\text {-C- }\end{array}$ \\
\hline Spike 4 onset & $\begin{array}{l}\text { Temporal Mid L } \\
\text {-DC- }\end{array}$ & $\begin{array}{l}\text { Temporal Mid L } \\
\text {-DC- }\end{array}$ & $\begin{array}{l}\text { Amygdala L, Hip- } \\
\text { pocampus L } \\
\text {-C- }\end{array}$ \\
\hline Spike 5 onset & $\begin{array}{l}\text { Cerebellum } 6 \text { L, } \\
\text { Cerebellum Crus } \\
1 \mathrm{~L} \\
\text {-DC- }\end{array}$ & $\begin{array}{l}\text { Temporal Mid L } \\
\text {-DC- }\end{array}$ & $\begin{array}{l}\text { Amygdala L, Hip- } \\
\text { pocampus L } \\
\text {-C- }\end{array}$ \\
\hline
\end{tabular}


Table 3: EEG source imaging results of five non-averaged spikes selected from the EEG recording of an epilepsy patient. The epileptic focus lies in the amygdalo-hippocampal area. The results were visualized at the spikes' peaks. For the maximum source activation, anatomical labels from the AAL atlas are shown. If the maximum of the estimated source activity falls within the resected region, the result is concordant, -C-, with the resected region; if the maximum does not fall within the resected region, the result is disconcordant, -DC-, with the resected region.

\begin{tabular}{|c|c|c|c|}
\hline Datasets & $\begin{array}{l}\text { Anatomical labels } \\
\text { of LORETA result }\end{array}$ & $\begin{array}{l}\text { Anatomical labels } \\
\text { of STKF result }\end{array}$ & $\begin{array}{l}\text { Anatomical labels } \\
\text { of RSTKF result }\end{array}$ \\
\hline Spike 1 peak & $\begin{array}{l}\mathrm{N} \backslash \mathrm{A} \\
-\mathrm{DC}-\end{array}$ & $\begin{array}{l}\mathrm{N} \backslash \mathrm{A} \\
-\mathrm{DC}-\end{array}$ & $\begin{array}{l}\text { Amygdala L, Hip- } \\
\text { pocampus L } \\
\text {-C- }\end{array}$ \\
\hline Spike 2 peak & $\begin{array}{l}\text { Temporal Mid L } \\
\text {-DC- }\end{array}$ & $\begin{array}{l}\text { Temporal Mid L } \\
\text {-DC- }\end{array}$ & $\begin{array}{l}\text { Amygdala L, Hip- } \\
\text { pocampus L } \\
\text {-C- }\end{array}$ \\
\hline Spike 3 peak & $\begin{array}{l}\text { Temporal Inf L } \\
\text {-DC- }\end{array}$ & $\begin{array}{l}\mathrm{N} \backslash \mathrm{A} \\
-\mathrm{DC}-\end{array}$ & $\begin{array}{l}\text { Amygdala L, Hip- } \\
\text { pocampus L } \\
\text {-C- }\end{array}$ \\
\hline Spike 4 peak & $\begin{array}{l}\text { Temporal Mid L } \\
\text {-DC- }\end{array}$ & $\begin{array}{l}\text { Temporal Inf L } \\
\text {-DC- }\end{array}$ & $\begin{array}{l}\text { Amygdala L, Hip- } \\
\text { pocampus L } \\
\text {-C- }\end{array}$ \\
\hline Spike 5 peak & $\begin{array}{l}\mathrm{N} \backslash \mathrm{A} \\
-\mathrm{DC}-\end{array}$ & $\begin{array}{l}\mathrm{N} \backslash \mathrm{A} \\
-\mathrm{DC}-\end{array}$ & $\begin{array}{l}\text { Amygdala L, Hip- } \\
\text { pocampus L } \\
\text {-C- }\end{array}$ \\
\hline
\end{tabular}

